\title{
Hierarchical Fibrillar Scaffolds Obtained by Non- conventional Layer-By-Layer Electrostatic Self-Assembly
}

\author{
Sara M. Oliveira, Tiago H. Silva, * Rui L. Reis, and João F. Mano
}

For the regeneration of hard tissues, the combination of synthetic and natural-origin materials arises as one of the best solutions to obtain 3D scaffolds with both adequate mechanical properties and similar glycosaminoglycan and proteoglycan type and architecture. ${ }^{[1]}$ The ability to introduce nano/micro structures inside scaffolds is of great interest as a way to achieve those cells' environments and mimic the hierarchical organization of natural tissues. The external scaffolds may provide the necessary mechanical integrity and the macro/micro organization of the overall structure and the inner nano/micro environment creates further attachment points for cell anchorage, and may provide distinct biochemical environments capable of controlling cell behaviour. With this purpose, micro/nano fibers produced by electrospinning have been combined with rapid prototyping and fiber-bonding techniques to produce scaffolds. ${ }^{[2]}$ However, this processing method has been hampered by limited and heterogeneous distributions of micro/nano fibers throughout the scaffold's bare structure. In fact, only surface modification was achieved; to produce scaffolds with internal nano/micro-structure, electrospinning needs to be done during bare scaffold preparation in a complex process that can lead to delamination of the final structure. Alternatively, freeze-drying of a polymeric structure inside a previously prepared scaffold may also be used to create a more native-like environment for cells; however, it is difficult to control the thickness and the morphology of this inner structure. ${ }^{[3,4]}$

The layer-by-layer (LbL) technique has been widely used to produce nanofilms for biomedical applications since its disclosure by Decher and co-workers ${ }^{[5]}$ based on a simple alternating deposition of polyanions and polycations, i.e. polyelectrolytes (PEs). The majority of the extracellular molecules and naturalorigin polymers can be used as PEs, with the ability to interact with complementary PEs not only by electrostatic attraction, but also through hydrogen bonding or hydrophobic interactions. Studies of LbL self-assembly in 2D have demonstrated that it is possible to control the surface chemistry, the surface

\section{S. M. Oliveira, Dr. T. H. Silva, Prof. R. L. Reis, \\ Dr. J. F. Mano \\ 3Bs Research Group-Biomaterials \\ Biodegradables and Biomimetics \\ AvePark, Zona Industrial da Gandra S. Claúdio do Barco \\ 4806-909 Caldas das Taipas - Guimarães, Portugal \\ E-mail: tiago.silva@dep.uminho.pt \\ S. M. Oliveira, Dr. T. H. Silva, Prof. R. L. Reis, Dr. J. F. Mano \\ ICVS/3B's - PT Government Associate Laboratory \\ Braga/Guimarães, Portugal}

DOI: $10.1002 /$ adhm.201200204 roughness and stiffness and, therefore, cell behaviour, by controlling the type and the number of bilayers. ${ }^{[6-13]}$ Moreover, one can also find in the literature studies where polyelectrolyte multilayers (PEMs) are used for surface modification of 3D scaffolds, aiming to improve cell-scaffold interactions, ${ }^{[14]}$ or to act as growth factor reservoirs. ${ }^{[15,16]}$ Additionally, using appropriate templates, highly porous scaffolds built entirely of nanostructured multilayers may be prepared. ${ }^{[17]}$

To our knowledge, the LbL technology has not yet been utilized to develop inner structures inside previously fabricated scaffolds. In the conventional dipping LbL approaches, nonreacted or weakly bonded polyelectrolyte should be removed throughout the washing steps in order to obtain stable polyelectrolyte multi-layered films and coatings. Herein, by conjugating non-conventional LbL self-assembly, based on incomplete washing steps to create small complexes, with ice crystal growth, it was possible to modify synthetic 3D scaffolds with natural-origin polymers, creating hierarchical and fibrillar structures in the interior of the scaffolds.

With these structures, one can increase the surface available area for cell growth and create a biochemical and structural environment similar to that found in the natural fibrillar extracellular matrix, while maintaining the mechanical properties of the bare scaffold. Some works on the production of hybrid structures, combining hydrogels or collagen, scaffolds and freeze-drying are found in the literature. ${ }^{[18,19]}$ However, those studies rely on the fibrous nature of collagen, not exhibited by most of the PE available, or are complex to control, and thus such approaches hardly create fibrillar structures, being mostly limited to lamellar ones. Other advantages of our approach may be pointed out: (1) no employment of toxic crosslinkers to stabilize the structures or improve the bonding with the scaffold; (2) virtually, the structures may be made using any polycation/ polyanion; (3) ability to create highly controlled delivery systems on the coatings and fibrillar structures.

In our group, the LbL technology has been used in different tissue engineering approaches, in particular based on the electrostatic self-assembly of alginate and chitosan PEs. ${ }^{[20-22]}$ Therefore, chitosan and alginate were the pair of PEs selected to achieve the modification of poly( $\varepsilon$-caprolactone) (PCL) scaffolds prepared by rapid prototyping rendering hierarchical fibrillar scaffolds, following the methodology illustrated in Scheme 1a. PCL scaffolds were first fabricated through a rapid prototyping technique, using a Bioplotter, with strut diameters of approximately $0.5 \mathrm{~mm}$ and 10 consecutive layers, each oriented at $90^{\circ}$ degrees with respect to the underlying layer. Morphological properties of 2D PEMs, such as: roughness, topography and thickness, are influenced by several build parameters, such 
a Methodology
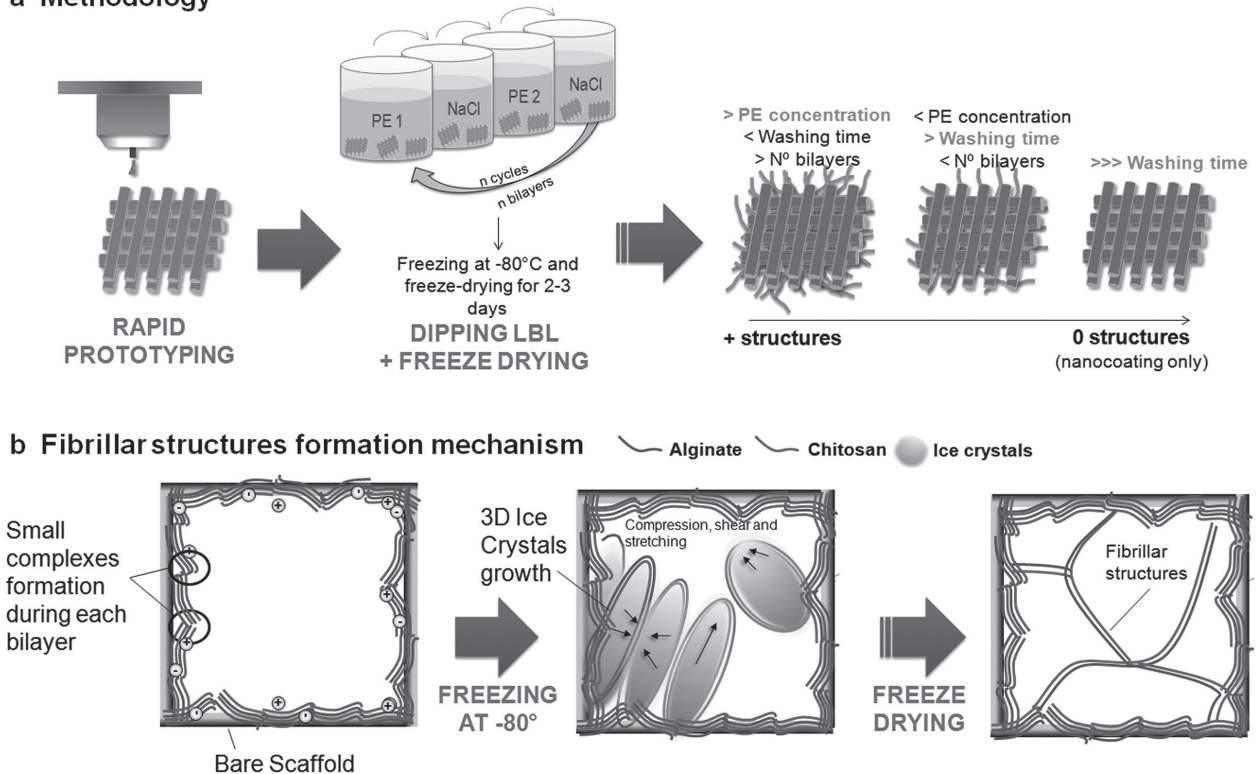

Scheme 1. (a) Steps for developing the hierarchical and hybrid 3D scaffolds. (b) Mechanism for the formation of the fibrillar structures.

as PE type, charge density, ionic strength, $\mathrm{pH}$, temperature, number of bilayers and washing time. When scaling up to assembly onto 3D porous substrates, parameters exhibiting a higher impact on the final structure are expected to be those that interfere most with the flow of the solutions throughout the pores, such as: PE concentration, dipping velocity, number of bilayers and surface wettability. In this sense, the influence of the number of bilayers, PE concentration, washing time and the PE nature of the outermost layer were assessed in this study. Briefly, a custom-made dipping robot was used to perform the LbL self-assembly by immersing diamine-modified PCL scaffolds: (1) in alginate solution during 10 minutes, (2) in a washing solution for 5 minutes, (3) in chitosan solution for 10 minutes, and (4) in a washing solution for 5 minutes. This process was repeated until the 15 PE double layers were attained, after which the scaffolds were washed several times with ultrapure water with agitation, and immediately frozen at $-80^{\circ} \mathrm{C}$ to let ice crystals to grow - Scheme $1 \mathrm{~b}$. The final structure was obtained after freeze-drying, thus producing the samples identified as $15 \mathrm{dL}$ (15 double layers). By changing one of the experimental conditions, as summarized in Table 1, other

Table 1. Nomenclature of the samples and respective LbL building up parameters.

\begin{tabular}{lcccc}
\hline Sample & No. bilayers & Outermost layer & $\begin{array}{c}\text { Washing } \\
{[\mathrm{min}]}\end{array}$ & $\begin{array}{c}\text { Conc. } \\
{\left[\mathrm{mg} \mathrm{ml}^{-1}\right]}\end{array}$ \\
\hline PCL & - & - & - & - \\
15dL & 15 & Chi & 5 & 0.5 \\
+ Conc & 15 & Chi & 5 & $\mathbf{5}$ \\
$30 \mathrm{dL}$ & $\mathbf{3 0}$ & Chi & 5 & 0.5 \\
+ Alg & 15 & Alg & 5 & 0.5 \\
Coat & 15 & Chi & $\mathbf{2 0 + 2 0}$ & 0.5 \\
\hline
\end{tabular}

groups of samples were produced: +Conc by increasing the concentration of PE solutions, 30dL by repeating the dipping cycle 30 times instead of only $15,+\mathrm{Alg}$ by depositing an additional alginate layer and Coat by increasing dramatically the duration of the washing steps.

The morphology of the bare and modified scaffolds was observed by scanning electron microscopy (SEM)-Figure 1. In addition, pores were observed in detail by stereomicroscopy, with respective images being depicted as shown in Figure S1 of the Supporting Information (SI). Moreover, the scaffolds were observed also by reflected microscopy with a $480 \mathrm{~nm}$ light source, since the high fluorescence intensity of chitosan at this wavelength allows the detection of chitosan on the structures. Chitosan was detected in all scaffolds except on the bare PCL. As it can be observed in the SEM micrographs in Figure 1 (longitudinal section) and in the other images (top view), the fibrillar structures are not only present in one layer/section of the scaffold, but also even in all the struts of the scaffold, vertical and horizontal. A distribution in xyz plans like this is not possible to obtain with hierarchical scaffolds employing electrospinning, where the fibers are deposited in just one xy plan, unless complex and low successful methodologies are employed, as discussed above. For the 15dL sample, used as reference, fibrillar structures were observed inside the dried scaffolds, ranging from nano to sub-micro scale. The density could be increased in different directions: mostly closer to the struts by increasing number of bilayers (30dL) or uniformly inside the pores by increasing PEs concentration (+Conc). When using higher washing times, such fibrillar structures were not observed; in these cases the PEM self-assembled just over the surface of the PCL struts constituting the scaffold, forming PE coatings (Coat). The formation of such fibrillar structures could then be attributed to the mechanism illustrated in Scheme 1b. With shorter washing steps, excess quantities of PEs remain inside the pores, giving rise to small complexes next to the pores wall; 


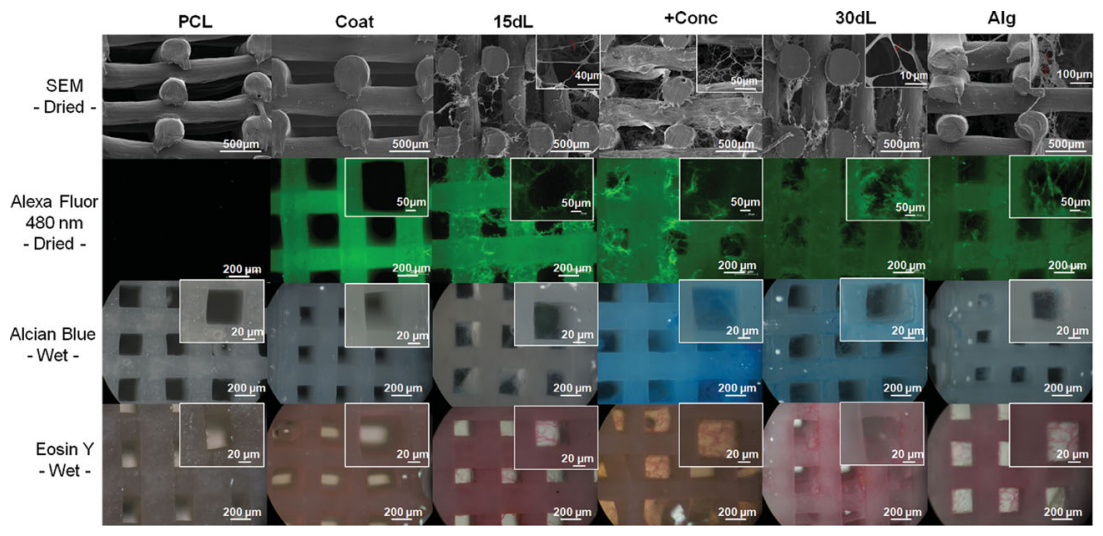

Figure 1. Representative images of the structures of the scaffolds (from top to bottom): SEM micrographs; images of the auto-fluorescence of chitosan using a transmitted- and reflectedlight microscope; stereomicroscope images of the Alcian blue staining; and stereomicroscope images of the eosin $Y$ staining.

those complexes progressively grow with the multilayer buildup. By using PEs, the structures became hydrophilic and water is entrapped inside the pores. Freezing the structures at $-80{ }^{\circ} \mathrm{C}$ triggers the formation of ice crystals that compress, shear and stretch the LbL constructs and small complexes, inducing the fibrillar-like morphology. In the case of higher concentration (+Conc), the viscosity of the PEs solutions was increased, limiting the efficiency of the washing step and enhancing the accumulation of remaining PEs molecules inside the volume of the pores, which promoted the development of more complexes in the whole volume of the scaffold. On the other hand, when longer washing steps (Coat) were used, no PE remain free inside the pores and thus no polyelectrolyte complexes were promoted besides the PEM coating the surface of the PCL struts. The scaffolds were also observed by stereomicroscope, after specific staining for each PE: Alcian blue for alginate (blue colour) and eosin Y for chitosan (pink, red colour); which colour intensiveness increases with the polymer content-Figure 1 . Significant blue and pink colours were observed on all scaffolds (except in PCL), not only on the surface but also on the fibrillar structures, thus showing the presence of alginate and chitosan on those scaffolds.

The morphology and 3D structure of the modified scaffolds were also observed by micro-computed tomography $(\mu \mathrm{CT})$-see Figure 2. Within the limited resolution of $\mu \mathrm{CT}$ scanner it was possible to observe some of the PE structures distributed throughout the pores of the scaffold, namely in the sample +Conc-Figure 2a down, in opposition to bare scaffold-Figure 2a top, where no such structures were observed. The poor definition of the fibrillar structures in the sample +Conc is attributed to its reduced dimensions, smaller than the resolution of a
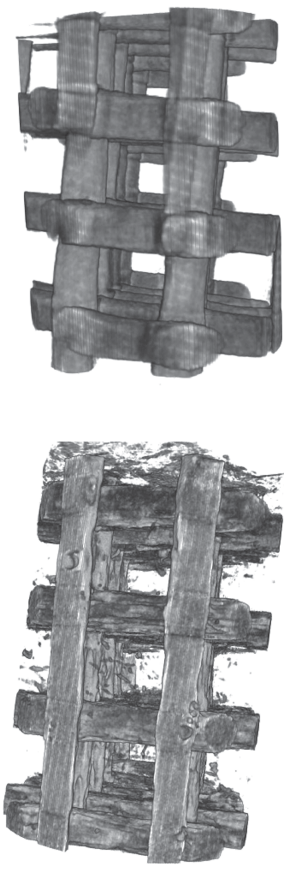

the $\mu \mathrm{CT}$ scanner. In fact, SEM micrographs showed that some of those features had thickness smaller than $1 \mu \mathrm{m}$. Besides influencing the morphology of the scaffolds, the effect of PEs assembly in other relevant properties was also assessed, namely on the water uptake and mechanical properties, known to influence also the cell behavior. Introducing PEM coatings or fibrillar structures in PCL scaffolds is expected to give them the ability to uptake aqueous solutions due to the increase in surface charge density and thus hydrophilicity. The water uptake property of the modified scaffolds was determined from the change of the weight upon immersion in phosphate-bufferd saline (PBS) solution, at $37{ }^{\circ} \mathrm{C}$; the obtained results are shown in Figure 2b. Comparing PCL with the +Conc sample, the average of the water uptake values were increased $40 \%$ on the latter, after reaching the equilibrium condition. The residual water uptake value evidenced by PCL sample is attributed to water retained inside the pores. Moreover, a faster water uptake of the +Conc was also observed, which is explained by the higher content and more uniform distribution of alginate and chitosan structures inside the scaffold's void volume. The analysis of the other samples has demonstrated that by controlling the concentration of the PEs and the number of bilayers, the swelling behaviour of the hybrid scaffolds may be controlled. This is an important property to b
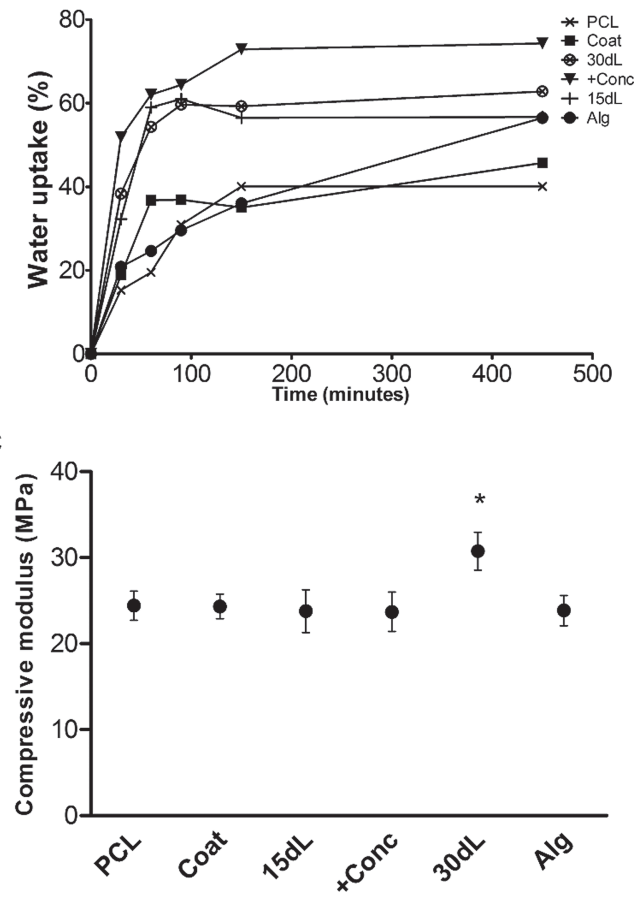

Figure 2. (a) $3 \mathrm{D}$ scan of $\mathrm{PCL}$ (top) and +Conc (bottom) samples, obtained by $\mu \mathrm{CT}$. (b) Wateruptake behaviour as function of the immersion time in PBS at $37^{\circ} \mathrm{C}$; (c) and compressive modulus of the studied scaffolds (* indicates all samples are statistically different from $30 \mathrm{dL}$; samples with normal distribution were tested using t-test, $p<0.065 ; n>5$ ). 


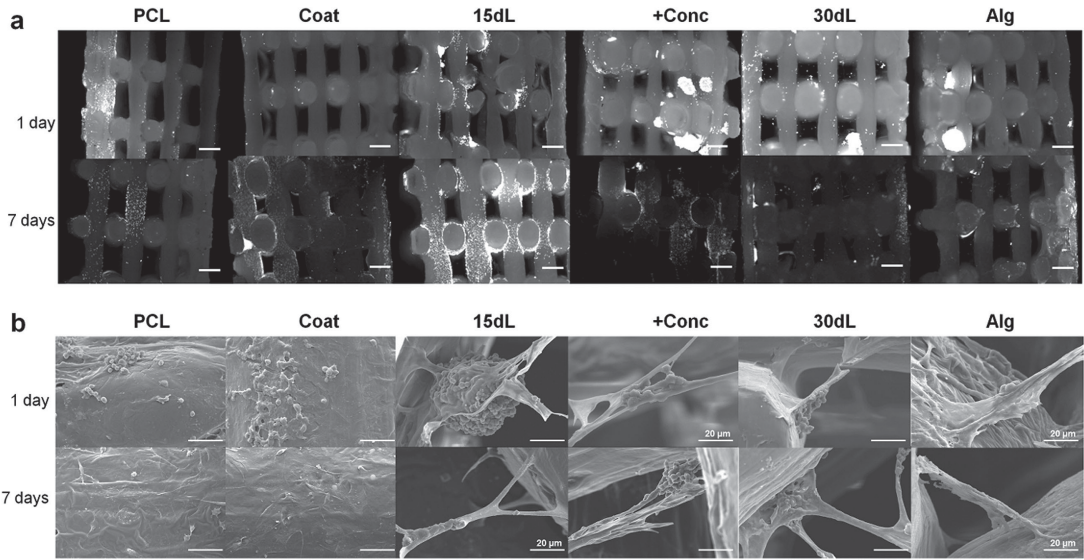

Figure 3. (a) Scaffold longitudinal central section images of the cell nucleus stained with DAPI after 1 and 7 days in culture (scale bar: $200 \mu \mathrm{m}$ ); (b) SEM micrographs showing SaOs-2 cell morphology and adhesion onto the surfaces or onto the fibrillar structures after 1 and 7 days in culture (scale bar: $50 \mu \mathrm{m}$, where not indicated).

develop reservoirs of ions or bioactive compounds localized on the PEs constructs, to be explored in future works. In order to verify if PEs assembly alters the mechanical properties of the dried scaffolds, uniaxial compression tests were performed on the cubic shape scaffolds. The compressive modulus for each case was determined from the stress-strain data and the results for each sample group are illustrated in Figure 2c. The compressive modulus remained unchanged despite the assembly of PEs, being approximately $24 \mathrm{MPa}$. An exception was observed after 30 cycles of alginate+chitosan assembly (30dL sample), whose value shifted statistical significantly to $30.8 \pm 5.4 \mathrm{MPa}$, comparing to all samples. This increase is attributed to a larger accumulation of PEs and structures mainly on the struts of the bare PCL scaffold than on the pores, thus reinforcing the whole 3D structure. In fact, when the density of such structures is higher but more uniformly distributed in whole pores volume, on +Conc sample, there was no significant increase in the compressive modulus. Therefore, it is possible to control the assembling conditions in order to create hybrid scaffolds, combing fibrillar structures made of natural-origin materials inside PCL scaffolds, without compromising the original mechanical properties.

The ultimate goal of the modification of scaffolds by PE assembly is to improve cell seeding efficiency and cell behavior. In order to assess the biological behavior of the PE modified scaffolds, their cytocompatibility was firstly evaluated, using SaOs-2 osteoblast-like cells, envisioning a bone tissue engineering application. Cells were cultured during 1 and 7 days for the cell distribution analysis by DAPI staining and SEM-Figure 3 and dsDNA and ALP activity quantification-Figure 4 . To further characterize distribution of viable cells, live/dead assay was also performed, with results being available in the SI, Figure S2. Cell attachment and adhesion occur within the first 24 hours in culture and are dictated by several surface properties including roughness, a

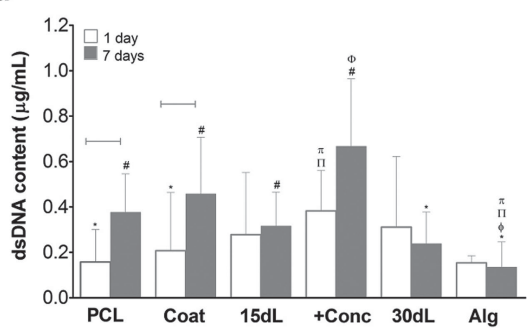

surface chemical groups, surface wettability, topography, protein adsorption, medium conditions and are also dependent on the cell type. ${ }^{[23]}$ Rapid prototyped scaffolds have highly controlled and reproducible architectures, but those 3D structures are commonly characterized by big open pores and smooth surfaces that reduce cell seeding efficiency. The introduction of alginate+chitosan structures on the PCL prototyped scaffolds acted as cell entrapment networks where SaOs-2 cells were able to adhere, thus increasing cell attachment and cell seeding efficiency, particularly on sample +Conc, as can be seen after DAPI staining-Figure 3a 1day. Those findings are supported by SEM images, showing cell attachment in fibrillar structures (Figure 3b) and dsDNA quantification results (Figure 4a), showing higher dsDNA quantity on the scaffolds exhibiting the fibrillar structures distributed in whole pore volume (+Conc) than on the ones where those fibrillar structures are more close to the walls (Coat, 15dL, 30dL, Alg samples). Alginates are known to lack mammalian cell-adhesive ligands, which are important to promote and regulate cellular interactions, and support low protein adsorption, ${ }^{[24,25]}$ which justifies the lowest dsDNA content observed for Alg sample (alginate as the outermost layer). The cationic nature of chitosan allows electrostatic interactions with anionic glycosaminoglycans (GAGs) and proteoglycans. ${ }^{[26,27]}$ Moreover, chitosan amine groups are known to increase the adsorption of proteins onto the PEM, creating more adhesive points for cells. ${ }^{[28]}$ However, when assembled with other PE, the amine groups which would bind to proteins or cells are not free. Consequently, cells have shown a tendency to form aggregates (Figure 3b 1 day) where the inner cells have lost viability (Figure S2 of the SI) and further come out of the scaffold after 7 days in culture (Figure 3b 7 days). The loss of cells upon formation of aggregates as a consequence of the low cell-adhesive groups on the alginate and chitosan has made statistically undetectable cell proliferation on the fibrillar scaffolds (Figure 4a). In future, polyelectrolytes able to enhance cell adhesion will be used to optimize the biological performance of the scaffolds. Nevertheless, in the majority of the conditions, SaOs- 2 cells were able to colonize whole longitudinal section, being present

Figure 4. (a) dsDNA content, and (b) ALP activity of SaOs-2 per unit of dsDNA after 1 and 7 days in culture. All significant differences are indicated: $\square$ - different from $15 \mathrm{dL}, \Phi$ - different from $30 \mathrm{dL}, \pi$ - different from PCL, $\Pi$ - different from Coat, $*$ - different from + Conc, \# - different from Alg (non-parametric Kruskal-Wallis and Dunn's post-hoc test were used, $p<0.05 ; n>6$ ). 
not only on the walls of the scaffolds but also in the fibrillar structures, as shown in Figure 3. When comparing the ALP activity results obtained with modified scaffolds with the ones obtained in tissue culture polystyrene (2D culture, positive control), it was observed that the introduction of fibrillar structures did not compromise the ALP activity of SaOs-2 cells after 1 day in culture, but on the contrary, ALP activity was improved, with exception of Alg sample, being the up-regulation more pronounced in +Conc sample. After 7 days in culture, ALP activity was generally stabilized around the value observed for the +Conc sample, with exception of the Coat sample, where no fibrillar features have been formed, which has shown significantly lower value of ALP activity. The positive effect of nanostructures on the enhancement of ALP activity has been pointed out previously in the literature, namely when using carbon nanotubes ${ }^{[29]}$ or PCL nanofiber meshes, ${ }^{[30]}$ supporting the attribution of a positive effect on cell seeding efficiency, as well as on cell activity and maturation, to the fibrillar structures created by PEs assembly.

In conclusion, the present work has revealed a successful methodology to create nano/micro fibrillar structures of alginate and chitosan inside 3D scaffolds, which may be extended to other types of PEs. By controlling the self-assembly parameters, namely washing time and PEs concentration, the density and distribution of these structures can be controlled. Although there is a lack of adhesive sites on alginate+chitosan structures, which can be overcome with the selection of other PEs, SaOs-2 cells seeding efficiency have been improved in sample +Conc, with cells being able to colonize the entire 3D scaffolds, including the introduced fibrillar structures, in 7 days, exhibiting also a significant up-regulated cell activity in day 1 . Thus, the introduction of such fibrillar features promises to play a role on the development of a new generation of hybrid scaffolds for tissue-engineering approaches, in particular ones containing an environment more identical to the fibrillar structure and nature of natural ECM, possessing at the same time a 3D structure able to support the mechanical requirements once implanted in vivo.

\section{Experimental Section}

Materials: PCL (Mw 70000 to 90 000), Chi (medium Mw), ethylenediamine and sodium alginate $(250 \mathrm{cP}, 2 \%), 2$-propanol, $\mathrm{NaHO}$; DMEM, FBS, ATB, trypsin-EDTA, and PBS were purchased from SigmaAldrich.

Fabrication of $P C L$ scaffolds: $P C L$ granules were inserted in the Bioplotter cartridge and heated up to $90^{\circ} \mathrm{C}$. Material was extruded by a 22C hypodermic needle with a strand size of $0.5 \mathrm{~mm}$ and layer thickness of $0.3 \mathrm{~mm}$ with struts aligned by $90^{\circ}$ in 10 consecutive layers.

Introduction of fibrillar structures: Scaffolds were cut with $0.5 \times 0.5 \mathrm{~cm}$ (widthxlength) and modified with $10 \%$ ethylenediamine in 2-propanol during 1 hour and $37{ }^{\circ} \mathrm{C}$. A custom-made robot was used to perform $\mathrm{LbL}$ assembly by immersing scaffolds first on alginate solution during 10 minutes, followed by the $\mathrm{NaCl}$ washing solution during 5 minutes, chitosan solution during 10 minutes and again washing solution during 5 minutes. This immersion sequence was repeated until completing 15 cycles-15dL samples. Using different washing time, polyelectrolyte solution concentration and number of cycles, new groups of samples were obtained, according to Table 1. After the LbL, scaffolds were washed under mild agitation in $\mathrm{NaCl}$ solution and ultrapure water, and then, frozen at $-80^{\circ} \mathrm{C}$. Final step consisted on the water removal by freeze-drying.
Characterization: Scaffolds were characterized by the observation of the polyelectrolytes after staining, $\mu \mathrm{CT}$, water uptake, and compression modulus. The water uptake percentage was calculated by the differences of weight between the dried and the wet scaffolds. Instron 4505 Universal Machine was used with a crosshead speed of $2 \mathrm{~mm} \mathrm{~min}{ }^{-1}$ in the compression testing. The compressive modulus was determined in the most linear region of the stress-strain graph and in the cases that the yield stress was not clear it was calculated as the stress at the intersection of a line drawn parallel to the linear region and intercepting the $x$-axis at 3 to $5 \%$ strain. Scaffolds were observed under stereomicroscope after staining with Eosin $Y$ (staining for chitosan) and Alcian Blue (staining for alginate) and under a light source of $480 \mathrm{~nm}$ under to observe the auto-fluorescent chitosan-based structures.

Cytocompatibility assessment: SaOs-2 were seeded on the developed scaffolds with a cellular density of 300000 cells $10 \mu \mathrm{L}^{-1}$ during 2.70 hours and then $1 \mathrm{~mL}$ of DMEM containing FBS. After 1 and 7 days in culture, cells were fixed using $2.5 \%$ formalin. Then, a number of samples were stained with DAPI $(1 \mathrm{mg} / \mathrm{ml})$ and observed under the reflected transmitted microscope with a light source of $358 \mathrm{~nm}$. Scaffolds with non-fixed cells were used to perform live/dead assay. dsDNA quantification was performed as indicated by the product provider (Quant-iT PicoGreen; Invitrogen). The ALP was evaluated using $p$-nitrophenol assay. Paranitrophenyl phosphate, which is colourless, is hydrolysed by alkaline phosphatase at $\mathrm{pH} 9.8$ and $37^{\circ} \mathrm{C}$ to form free $p$-nitrophenol, which is coloured yellow. The reaction is stopped by addition of $\mathrm{NaOH}$ and the absorbance read at $405 \mathrm{~nm}$. For SEM observation under Leica Cambridge microscope, cells were fixed with $2.5 \%$ glutaraldehyde and the scaffolds dehydrated by an increasing concentration sequence of ethanol solutions.

\section{Supporting Information}

Supporting Information is available from the Wiley Online Library or from the author.

\section{Acknowledgements}

This work was partially supported by the European Union/ EFDR through the POCTEP project 0330_IBEROMARE_1_P. Portuguese Foundation for Science and Technology is gratefully acknowledged for fellowships of S.M.O. (SFRH/BD/70107/2010) and T.H.S. (SFRH/BPD/34704/2007). The authors wish to acknowledge J. Miguel Oliveira for assistance with micro-CT measurements.

Received: June 19, 2012

Revised: August 21, 2012

Published online:

[1] G. Chen, T. Sato, T. Ushida, N. Ochiai, T. Tateishi, Tissue Eng. 2004, 10, 323.

[2] S. H. Park, T. G. Kim, H. C. Kim, D. Y. Yang, T. G. Park, Acta Biomater. 2008, 4, 1198.

[3] J. F. Mano, G. Hungerford, J. L. Gómez Ribelles, Materials Science and Engineering: C 2008, 28, 1356.

[4] J. Antunes, J. Oliveira, R. Reis, J. Soria, J. Gómez-Ribelles, J. Mano, J Biomed. Mater. Res. A 2010, 94, 856.

[5] G. Decher, J. D. Hong, J. Schmitt, Thin Solid Films 1992, $210,831$.

[6] M. Zenobi-Wong, R. F. Mhanna, J. Voros, Biomacromolecules 2011, 12, 609.

[7] P. R. Van Tassel, J. A. Phelps, S. Morisse, M. Hindie, M. C. Degat E. Pauthe, Langmuir 2011, 27, 1123.

[8] C. Picart, T. Boudou, T. Crouzier, C. Nicolas, K. Ren, Macromol. Biosci. 2011, 11, 77. 
[9] T. Groth, M. S. Niepel, D. Peschel, Int. J. Artif. Organs 2011, 34, 185.

[10] B. H. Choi, Y. S. Choi, H. J. Cha, Abstr. Pap. Am. Chem. S. 2011, 241.

[11] E. L. Chaikof', J. T. Wilson, W. X. Cui, V. Kozovskaya, E. Kharlampieva, D. Pan, Z. Qu, V. R. Krishnamurthy, J. Mets, V. Kumar, J. Wen, Y. H. Song, V. V. Tsukruk, J. Am. Chem. Soc. 2011, 133, 7054.

[12] J. H. Y. Ting, M. R. Haas, S. M. Valenzuela, D. K. Martin, let. Nanobiotechnol. 2010, 4, 77.

[13] C. Picart, K. F. Ren, L. Fourel, C. G. Rouviere, C. Albiges-Rizo, Acta Biomater. 2010, 6, 4238

[14] Y. H. Gong, Y. B. Zhu, Y. X. Liu, Z. W. Ma, C. Y. Gao, J. C. Shen, Acta Biomater. 2007, 3, 677.

[15] M. L. Macdonald, R. E. Samuel, N. J. Shah, R. F. Padera, Y. M. Beben, P. T. Hammond, Biomaterials 2011, 32, 1446.

[16] T. Crouzier, K. Ren, C. Nicolas, C. Roy, C. Picart, Small 2009, 5, 598.

[17] P. Sher, C. A. Custódio, J. F. Mano, Small 2010, 6, 2644.

[18] G. P. Chen, T. Sato, T. Ushida, R. Hirochika, Y. Shirasaki, N. Ochiai, T. Tateishi, J. Biomed. Mater. Res. A 2003, 67A, 1170.

[19] W. D. Dai, N. Kawazoe, X. T. Lin, J. Dong, G. P. Chen, Biomaterials 2010, 31, 2141.
[20] N. M. Alves, C. Picart, J. F. Mano, Macromol. Biosci. 2009, 9, 776.

[21] G. V. Martins, E. G. Merino, J. F. Mano, N. M. Alves, Macromol. Biosci. 2010, 10, 1444.

[22] E. S. Miranda, T. H. Silva, R. L. Reis, J. F. Mano, Tissue Eng. Pt A 2011, 17, 2663.

[23] N. M. Alves, I. Pashkuleva, R. L. Reis, J. F. Mano, Small 2010, 6, 2208

[24] K. Y. Lee, D. J. Mooney, Prog Polym Sci 2012, 37, 106.

[25] J. A. Rowley, G. Madlambayan, D. J. Mooney, Biomaterials 1999, 20, 45.

[26] A. R. Costa-Pinto, R. L. Reis, N. M. Neves, Tissue Eng. Part B-Re 2011, 17, 331.

[27] T. W. L. Groeneveld, M. Oroszlan, R. T. Owens, M. C. Faber-Krol, A. C. Bakker, G. J. Arlaud, D. J. McQuillan, U. Kishore, M. R. Daha, A. Roos, J. Immunol. 2005, 175, 4715.

[28] H. G. Xie, X. X. Li, G. J. Lv, W. Y. Xie, J. Zhu, T. Luxbacher, R. Ma, X. J. Ma, J. Biomed. Mater. Res. A 2010, 92A, 1357.

[29] X. M. Li, H. Gao, M. Uo, Y. Sato, T. Akasaka, S. Abe, Q. L. Feng, F. Z. Cui, F. Watari, Biomed. Mater. 2009, 4.

[30] P. Wutticharoenmongkol, N. Sanchavanakit, P. Pavasant, P. Supaphol, J Nanosci. Nanotechno. 2006, 6, 514. 